\title{
Effectiveness of Reversible Contraception in Dog Population Management*
}

\author{
Oswaldo Santos Baquero, Ana Pérola Drulla Brandão, Marcos Amaku \& Fernando Ferreira
}

\begin{abstract}
Background: Dog fertility depends on human-influenced factors such as sterilization. Uncontrolled fertility can result in unwanted births and overpopulation, which causes problems of public health and animal welfare. Surgical sterilization has been the traditional means of reproduction control but its cost and time can be prohibitive for mass sterilization programs. Non-surgical sterilization alternatives exist, but most of them are reversible and their effectiveness as a population management tool is unknown. To better understand the consequences of reversible contraception, the fertility dynamics was modeled in a hypothetical dog population in a steady-state condition.

Materials, Methods \& Results: The effect of reversible contraception was simulated using a coupled system of ordinary differential equations. A hypothetical steady-state population of 1000 animals was considered. It was formed by two compartments, one of fertile dogs and the other of infertile dogs. Natality compensated for a fraction of mortality, and the immigration rate compensated for the remaining fraction. The group of immigrant dogs was composed of fertile and infgertile dogs. The dog flow between compartments was given by both the contraception and fertility recovery rate. It was assumed that fertility reversibility in animals of the immigrant group was equal to that of animals already present in the population. Global sensitivities were calculated to assess the uncertainties of fertility dynamics associated with estimation of parameters. In addition, the local sensitivities were calculated to assess the influence of each parameter on fertility dynamics. The treatment effectiveness were expressed in terms of the total number of dogs treated for 20 years by taking a given irreversible contraception rate divided by the total number of dogs treated during the same period, and using the corresponding reversible contraception rate. The global sensitivity analysis consistently indicated a reduction in the number of fertile dogs. The local sensitivity analysis indicated that contraception rate was the most influential parameter, followed by the fertility recovery rate. The fraction of mortality compensated by natality was more influential than the fraction of infertile immigrants. Simulated scenarios indicated that the higher the contraception rate, the greater the difference between the effects of different fertility recovery rates. Variations in the proportion of infertile immigrants minimally changed the number of fertile dogs and the accumulated number of treated dogs. The increase in the fertility recovery rate caused effectiveness to decrease, especially when contraception rates were higher.

Discussion: In certain scenarios, reversible contraception can be a viable option for reproduction control. Evaluation of effectiveness of the reversible contraception showed both the importance of duration of the contraceptive effect and the interaction between the contraception and fertility recovery rates. Although the contraception rate is the main determinant of population fertility dynamics, the fertility recovery rate modulates the effect of contraception and determines its viability. Reversible contraception is a viable alternative when loss in effectiveness is compensated by a reduction in costs and ease of application of contraceptive treatments. The lower the contraception rate, the higher the similarity between the effects of reversible and irreversible contraception.
\end{abstract}

Keywords: sterilization, pets, reproduction control.

*Article based on a Dissertation submitted by the first author in partial fulfillment of the requirements for the PhD Degree. Departamento de Medicina Veterinária Preventiva e Saúde Animal (VPS), Faculdade de Medicina Veterinária e Zootecnia (FMVZ), Universidade de São Paulo (USP), São Paulo, SP, Brazil. CORRESPONDENCE: O. Santos Baquero [baquero@usp.br - Fax: +55 (11) 3091-7928]. Av. Prof. Dr. Orlando Marques de Paiva n. 87. CEP 05508-270 São Paulo, SP, Brazil. 


\section{INTRODUCTION}

Availability of resources and a good health status favor fecundity (reproductive potential) of dogs. However, fertility (actual reproductive performance) also depends on human-influenced factors such as sterilization, copulation prevention or pregnancy interruption [5,9].

Uncontrolled fertility can result in unwanted births and overpopulation, which causes problems of public health and animal welfare [17]. Reproduction control is one of the strategies for population management [7], which contributes to prevent abandonment $[11,15]$ and intervenes directly in unwanted births. Moreover, sterilization can change dog behaviors associated with abandonment and contribute to reduce injuries due to biting $[8,12,13]$.

The long-term effects of reproduction control are similar to those of euthanasia [1]. However, reproduction control is more ethical and is in agreement with the practices of responsible ownership as recommended by the OIE [19].

Surgical sterilization has been the traditional means of reproduction control [10], but its cost and time can be prohibitive for mass sterilization programs [9]. Non-surgical alternatives include hormonal treatments, immunocontraceptive vaccines, intratesticular or intraepididymal injections and mechanical methods [9]. For population management, safe and effective alternatives, which do not cause adverse effects or irreversible infertility, are preferable [14]. In most of the alternatives mentioned above, the effects are reversible, but their effectiveness as a population management tool is unknown. To better understand the effect of reversible contraception, the fertility dynamics was modeled in a hypothetical dog population in a steadystate condition.

\section{MATERIALS AND METHODS}

\section{Model}

The effect of reversible contraception was simulated using a coupled system of ordinary differential equations. A hypothetical steady-state population was considered. It was formed by two compartments, one of fertile $(n)$ and the other of infertile $(g)$ dogs (Figure 1). The natality compensated for a fraction $(r)$ of the mortality ( $m=$ mortality rate), and immigration compensated for the remaining fraction $(1-r)$. Among immigrant dogs, a fraction was of infertile $(z)$ and the remaining of fertile dogs $(1-z)$. The dog flow from compartment $n$ to $g$ was given by the contraception rate $(s)$, and the counter flow from compartment $g$ to $n$ by the fertility recovery rate $(f)$. Fertility reversibility in immigrant dogs was assumed to be equal to that of animals already in the population. In order to simplify the model, rate $f$ was applied to these immigrants as soon as they entered the population. The initial population was of 950 fertile and 50 infertile dogs, and all rates were constant.

$$
\begin{gathered}
\frac{d n}{d t}=r m(n+g)+(1-r) m(1-z)(n+g)+f g-(m+s) n \\
\frac{d g}{d t}=(1-r) m z(n+g)+s n-(m+f) g
\end{gathered}
$$

\section{Estimation of parameters}

Table 1 shows the values for the initial conditions and parameters. To keep the total population size constant, the source of immigration q was assumed to be equal to $n+g$.

\section{Global sensitivity}

Each parameter was simultaneously varied in an interval whose lower and upper limits were $10 \%$ lower and higher than the point estimate, respectively. The Latin hypercube algorithm [18] was used to sample the parameters of their respective intervals, in each one of the 100 population dynamics simulations.

\section{Local sensitivity}

In order to compare the influence of each parameter on both the total number of fertile dogs and accumulated number of treated dogs, sensitivity functions [4] were used. Thus, the sensitivity matrix $(t \times m)$ was defined as

$$
V=\frac{\partial x(\theta)}{\partial \boldsymbol{\theta}^{\mathrm{T}}} \mid \theta=\theta 0
$$

in which $\boldsymbol{\theta}=\left[q_{1} ; q_{2} ; \ldots ; q_{m}\right]^{T}$ was the parameter vector and $\boldsymbol{x}(\boldsymbol{\theta})=\left[x_{1}(\boldsymbol{\theta}) ; x_{2}(\boldsymbol{\theta}) ; \ldots ; x_{t}(\theta)\right]^{T}$ was the vector with the total fertile dogs or accumulated number of dogs treated in times $1 ; 2 ; \ldots ; t$. In order that sensitivities were free of scale, the scaled matrix $S=\left\{s_{i j}\right\}$ was used,

$$
s_{i j}=v_{i j} \frac{\theta j}{S C_{i}}
$$

where $v_{i j}$ denotes an element of $\mathbf{V}, \Delta \boldsymbol{\theta}_{j}$ is the interval in which $\theta_{j}$ varies (approximated by finite differences) and $S C_{i}$ is the scale factor defined as equal to the parameter value. The sensitivities were characterized with the L1 norm of the columns $s_{j}$. 


$$
L 1=\sqrt{\frac{1}{n} \sum_{i=1}^{n} s^{2 i j}}
$$

\section{Simulated scenarios}

Scenarios were built by combining variations in the contraception $(0.05,0.2$, and 0.4 year$1)$, fertility recovery rates $(0,0.01, \ldots, 0.999$, and 1 year-1) and infertile immigrant fraction $(0.05,0.2$, and 0.4) while maintaining the other parameters constant.

\section{Effectiveness of reversible contraception}

Effectiveness was expressed in terms of the total number of dogs treated for 20 years by taking a given irreversible contraception rate divided by the total number of dogs treated during the same period, and used a corresponding reversible contraception rate.

\section{Software}

All analyses were performed in the R software 3.1.2 [16] using the capm 0.8.0 package, which is a package for companion animal population management [2].

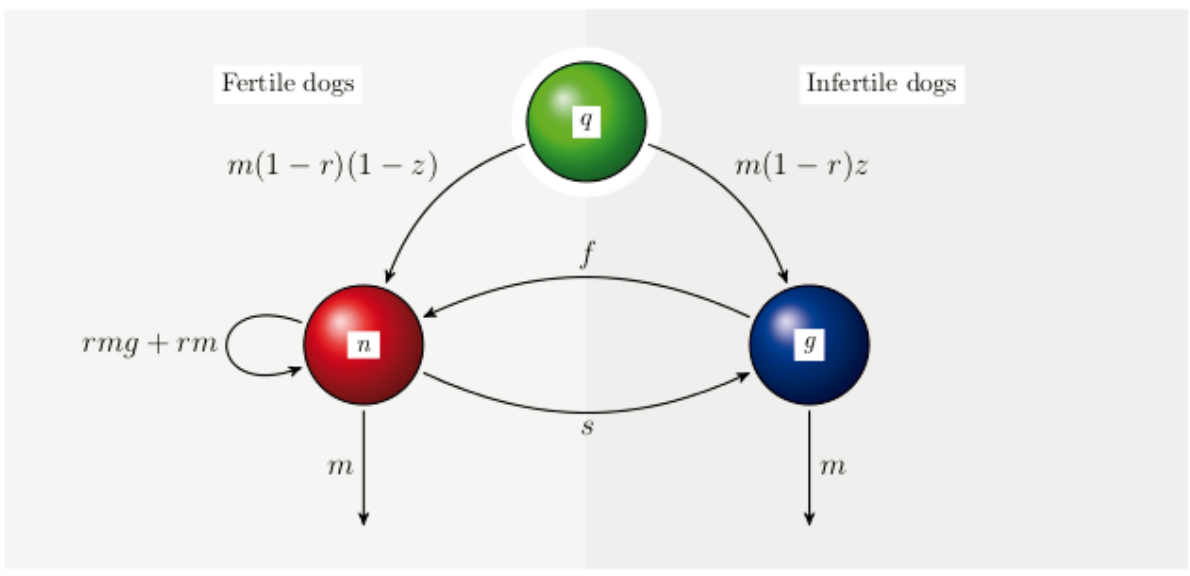

Figure 1. Compartmental model of a hypothetical dog population receiving contraceptive treatments. $n$ : fertile dogs; $g$ : infertile dogs; $q$ : immigration source $(q=\mathrm{n}+g) ; m$ : mortality rate; $r$ : fraction of mortality compensated by natality; $s$ : contraception rate; $f$ : fertility recovery rate; $z$ : fraction of infertile immigrants.

Table 1. Parameters and initial conditions in the model of a hypothetical dog population receiving contraceptive treatments.

\begin{tabular}{ccc}
\hline & Values & Description \\
\hline Initial conditions & & \\
$\mathrm{n}$ & 950 & Fertile dogs \\
$\mathrm{g}$ & 50 & Infertile dogs \\
Parameters & & \\
$\mathrm{m}^{*}$ & $1 / 6$ & Mortality rate $\left(\right.$ year $\left.^{-1}\right)$ \\
$\mathrm{s}$ & 0.2 & Contraception rate $\left(\right.$ year $\left.^{-1}\right)$ \\
$\mathrm{f}$ & 0.5 & Fertility recovery rate $\left(\mathrm{year}^{-1}\right)$ \\
$\mathrm{z}$ & 0.2 & Fraction of infertile immigrants \\
$\mathrm{r}$ & 0.8 & Fraction of mortality compensated by natality \\
\hline
\end{tabular}

*Inverse of life expectancy of female dogs. Source: Fernando Ferreira. 2009. [6] 


\section{RESULTS}

\section{Global sensitivity}

The global sensitivity analysis consistently indicated a reduction in the number of fertile dogs. After 6 years, the minimum and maximum values and the standard deviation stabilized around 733, 792, and 13, respectively (Figure 2).

\section{Local sensitivity}

The contraception rate was the most influential parameter, followed by the fertility recovery rate. The fraction of the mortality compensated by natality was more influent than the fraction of infertile immigrants (Figure 3).

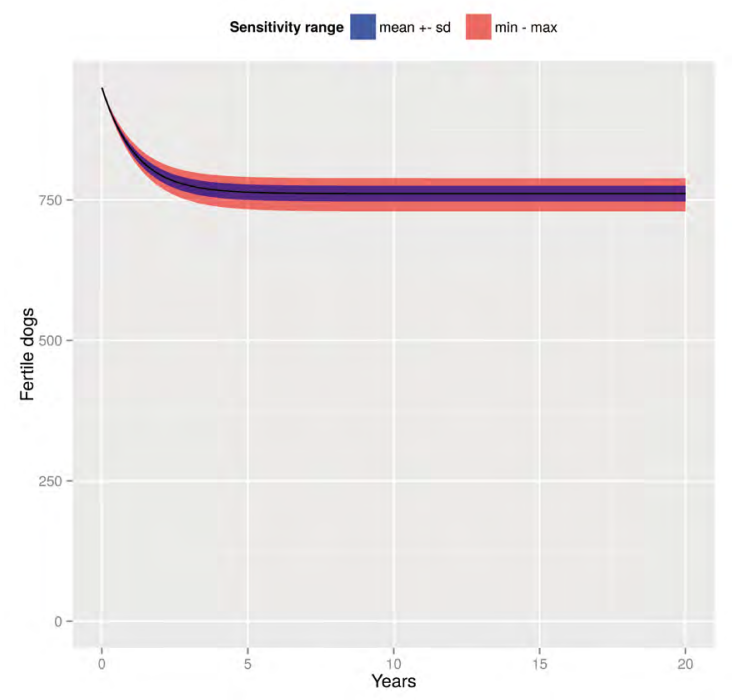

Figure 2. Global sensitivity of the total number of fertile dogs.

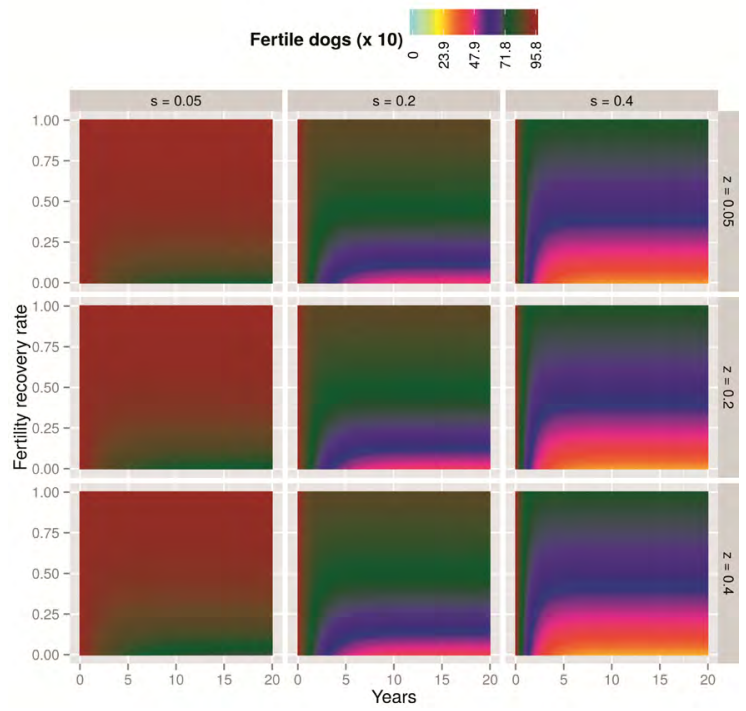

Figure 4. Total number of fertile dogs along time as a function of the fertility recovery rate, contraception rate (s), and fertile immigrant fraction (z). Rate unit: year-1.

\section{Simulated scenarios}

The higher the contraception rate, the greater the difference between the effects of different fertility recovery rates. Variations in the proportion of infertile immigrants minimally changed the number of fertile dogs and the accumulated number of treated dogs (Figures 4 and 5).

\section{Effectiveness of reversible contraception}

Effectiveness varied between 0 and 1 and, as defined, irreversible contraception effectiveness was equal to 1 , the maximum effectiveness. The increase in the fertility recovery rate decreased the effectiveness of the different contraception rates, especially the highest ones (Figure 6).

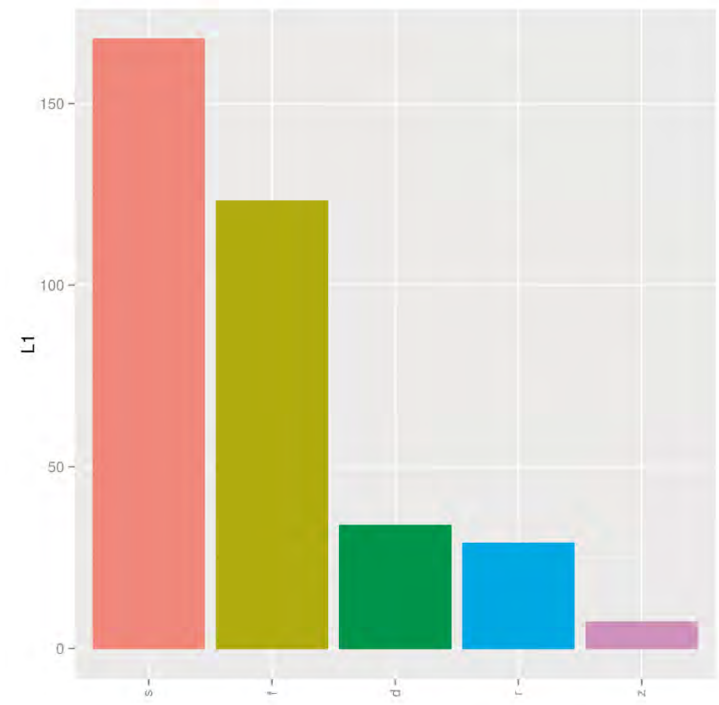

Figure 3. Local sensitivity of the total number of fertile dogs.

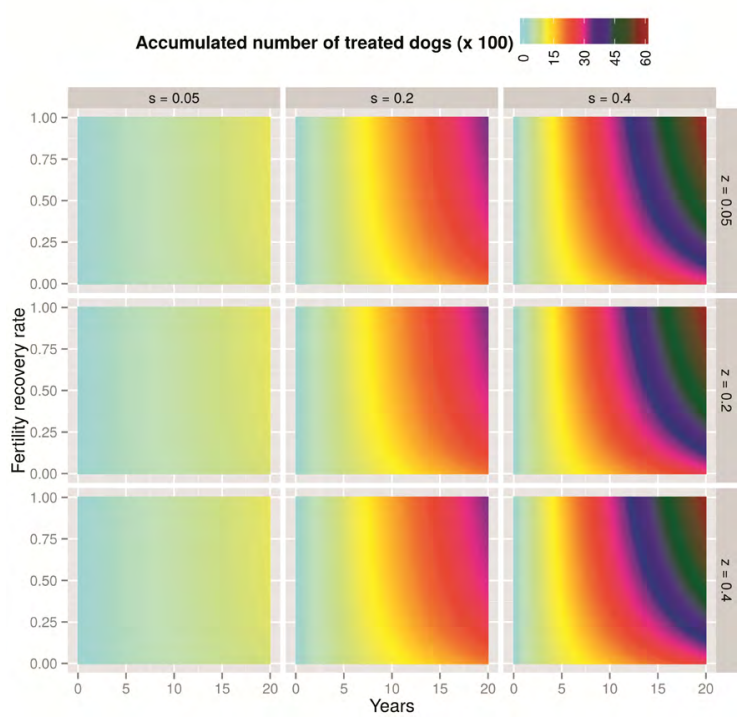

Figure 5. Accumulated number of dogs treated over time, as a function of the fertility recovery rate, contraception rate (s), and fertile immigrant fraction (z). Rate unit: year-1. 


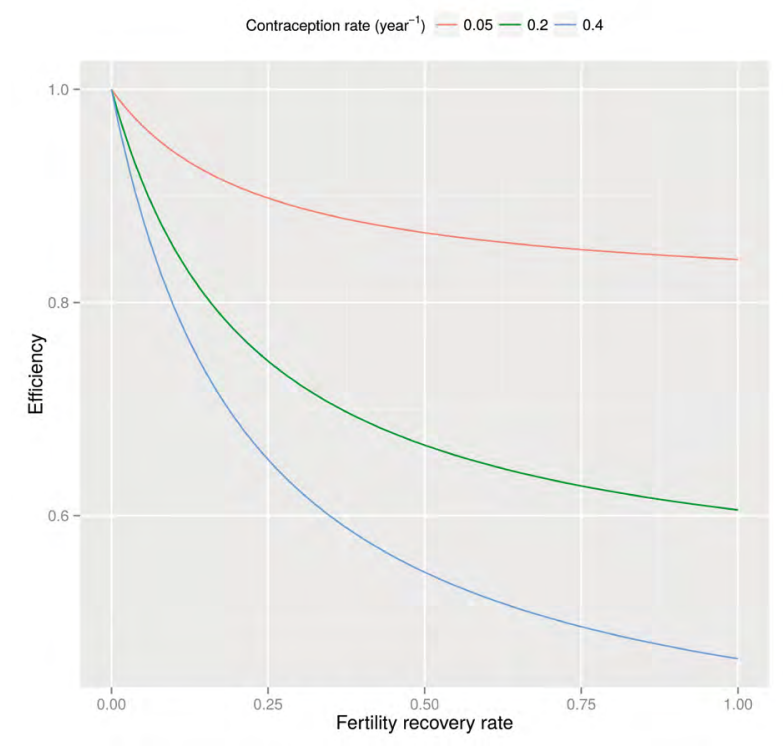

Figure 6. Effectiveness of reversible contraception for different contraception rates.

\section{DISCUSSION}

In certain scenarios, reversible contraception can be a viable option for reproduction control. Although the contraception rate is the main determinant of population fertility dynamics, the fertility recovery rate modulates the effect of contraception and determines their viability.

In steady-state populations, mortality and emigration are compensated by natality and immigration. In the model implemented in the study, the mortality was compensated both by natality and immigration. Although this can be interpreted as a scenario in which emigration does not occur, it can also be interpreted as a scenario with emigration which is implicitly compensated by natality and immigration, without changing the fraction of fertile dogs. In this sense, these rates, although unspecified, are higher than the fractions of mortality rate that they compensated. Not specifying the emigration, immigration, and natality rates was a simplification strategy. It allowed modeling an open population in scenarios conditioned by different fractions of infertile immigrants, and inclusion of these three parameters was not required.

Evaluation of effectiveness of the reversible contraception showed both the importance of duration of the contraceptive effect, and the interaction between the contraception and fertility recovery rates. The lower the contraception rate, the higher the similarity between the effects of reversible and irreversible contraception.
Reversible contraception may be feasible when loss in effectiveness is compensated by cost and ease of implementation, and such situation is favored as the treatment implementation and fertility recovery rates are lower.

The effectiveness of reversible contraception was given by constant rates. Thus, discontinuation of the reproduction control of a given number of dogs does not affect effectiveness if the same number of fertile dogs begins contraception treatment simultaneously. The difficulty to maintain and verify this condition in practice is an additional reason in favor of methods with a lower fertility recovery rate.

Given that the population simulated always remained at steady-state, reproduction control never caused a decrease in the population size. Even so, the effects of different contraception rates were compared and the effect they exerted on the population fertility dynamics was verified. As a previous study [3], such an approach shows that decrease in the population size is not required to define effectiveness of the reproduction control.

\section{CONCLUSIONS}

Reversible contraception is a viable alternative when loss in effectiveness is compensated by a reduction in costs and ease of application of contraception treatments. The lower the contraception rate, the higher the similarity between the effects of reversible and irreversible contraception. 
Funding. The authors acknowledge the funding given by FAPESP institution to this research.
Declaration of interest. The authors report no conflicts of interest. They wrote the manuscript and are responsible for its content.

\section{REFERENCES}

1 Amaku M., Dias R.A. \& Ferreira F. 2010. Dynamics and Control of Stray Dog Populations. Mathematical Population Studies. 17(2): 69-78.

2 Baquero O.S., Amaku M. \& Ferreira F. 2015. capm: an R package for Companion Animal Population Management. URL http://oswaldosantos.github.io/capm.

3 Baquero O.S., Akamine L.A., Amaku M. \& Ferreira F. 2016. Defining priorities for dog population management through mathematical modeling. Preventive Veterinary Medicine. (123): 121-127.

4 Brun R., Reichert P. \& Künsch H.R. 2001. Practical identifiability analysis of large environmental simulation models. Water Resources Research. 37(4): 1015-1030.

5 Eilts B.E. 2002. Pregnancy termination in the bitch and queen. Clinical Techniques in Small Animal Practice. 17(3): 116-123.

6 Ferreira F. 2009. Efeito da esterilização no controle de populações de cães. 38f. São Paulo, SP. Tese (Livre Docência) - Faculdade de Medicina Veterinária e Zootecnia, Universidade de São Paulo.

7 Garcia R., Calderón N. \& Ferreira F. 2012. Consolidação de diretrizes internacionais de manejo de populações caninas em áreas urbanas e proposta de indicadores para seu gerenciamento. Revista Panamericana de Salud Publica. 32(2): 140-144.

8 Gershman K.A., Sacks J.J. \& Wright J.C. 1994. Which dogs bite? A case-control study of risk factors. Pediatrics. 93(6): 913-917.

9 Kutzler M. \& Wood A. 2006. Non-surgical methods of contraception and sterilization. Theriogenology. 66(3): 514-525.

10 Looney A., Bohling M.W., Bushby P.A., Howe L.M., Griffin B., Levy J.K., Eddlestone S.M., Weedon J.R., Appel L.D., Rigdon-Brestle K., Ferguson N.J., Sweeney D.J., Tyson K.A., Voors A.H., White S.C., Wilford C.L., Farrell K.A., Jefferson E.P., Moyer M.R., Newbury S.P., Saxton M.A \& Scarlett J.M. 2008. The Association of Shelter Veterinarians veterinary medical care guidelines for spay-neuter programs. Journal of the American Veterinary Medical Association. 233(1): 74-86.

11 Marston L.C., Bennett P.C. \& Coleman G.J. 2004. What happens to shelter dogs? An analysis of data for 1 year from three Australian shelters. Journal of Applied Animal Welfare Science. 7(1): 27-47.

12 Messam L.L.M., Kass P.H., Chomel B.B. \& Hart L.A. 2008. The human-canine environment: a risk factor for nonplay bites? The Veterinary Journal. 177(2): 205-215.

13 Neilson J., Eckstein R. \& Hart B. 1997. Effects of castration on problem behaviors in male dogs with reference to age and duration of behavior. Journal of the American Veterinary Medical Association. 211(2): 180-182.

14 Oliveira E.C.S., Moura M.R.P., de Sá M.J., Silva V.A., Kastelic J.P., Douglas R.H. \& Marques A.P. 2012. Permanent contraception of dogs induced with intratesticular injection of a Zinc Gluconate-based solution. Theriogenology. 77(6): 1056-1063.

15 Patronek G.J., Glickman L.T., Beck A.M., McCabe G.P. \& Ecker C. 1996. Risk factors for relinquishment of dogs to an animal shelter. Journal of the American Veterinary Medical Association. 209(3): 572-581.

16 R Core Team. 2015. R: A Language and Environment for Statistical Computing. R Foundation for Statistical Computing, Vienna, Austria. URL http://www.R-project.org/. [Accessed online July 2015].

17 Slater M.R. 2001. The role of veterinary epidemiology in the study of free-roaming dogs and cats. Preventive Veterinary Medicine. 48(4): 273-286.

18 Soetaert K. \& Petzoldt T. 2010. Inverse modelling, sensitivity and Monte Carlo analysis in R using package FME. Journal of Statistical Software. 33: 1-28.

19 World Organization for Animal Health. 2010. Stray dog population control. Terrestrial Animal Health Code. Paris: OIE. Disponível em: <http://www.oie.int/index.php?id=169\&L=0\&htmfile=chapitre_aw_stray_dog.htm>. [Accessed online August 2015]. 